\title{
Anhang I: Studieninformation
}

\section{Studie: (Aus)Sicht medizinischer Akteure auf die Versorgung schwerstkranker und sterbender Menschen im Krankenhaus}

Das Krankenhaus ist mit ca. 50 \% Sterbeort Nummer eins - in Deutschland wie in anderen Industrienationen. Betrachtet man die demografische und medizinische Entwicklung, werden zukünftig trotz der gesundheitspolitischen Stärkung ambulanter palliativmedizinischer Versorgungsstrukturen sogar mehr Patienten mit fortgeschrittenen Krebserkrankungen oder anderen chronischen progredient verlaufenden Erkrankungen akut stationär aufgenommen werden. Oft wegen unbeherrschbarer Schmerzen, anderen gravierenden Symptomen oder wegen Problemen in der häuslichen Versorgung. Damit geht es zukünftig weniger um das $\boldsymbol{o b}$ des Sterbens in Institutionen, sondern um das Wie?

Ausgangspunkt des von der Berliner Krebsgesellschaft (BKG) geförderten Forschungsprojektes ist die identifizierte Differenz der programmatischen Überlegungen der Charta zur Betreuung schwerstkranker und sterbender Menschen und der Handlungspraxis eines Akutkrankenhauses der Maximalversorgung. Gesundheitspolitischen Forderungen steht eine konzeptionelle und habituelle Unsicherheit der medizinischen Akteure aus der Praxis gegenüber, wie Versorgungsstrukturen aussehen könnten, die der anspruchsvollen Forderung nach einer Integration palliativer Angebote quer zu allen medizinischen Fachdisziplinen im Krankenhaus nachkommt.

Im Rahmen der Studie befragen wir Ärzte verschiedener Fachdisziplinen in Experteninterviews bezüglich ihrer Erfahrungen in der Behandlung schwerstkranker und sterbender Menschen in der stationären Versorgung. Ihre Erfahrungsberichte aus der Praxis liefern wichtige Hinweise für die Entwicklung von Strukturen und Empfehlungen, die unterstützend bei der Behandlung dieser Patientengruppe ist.

Ziele der Studie und praktische Relevanz:

- Exploration der Erfahrungen von Ärzten verschiedener Fachdisziplinen in der Versorgung schwerkranker und sterbender Menschen im Krankenhaus

- Analyse der strukturellen und personellen Ressourcen im Forschungsfeld

- Beteiligung der medizinischen Akteure bei der Entwicklung von Empfehlungen für die Versorgung schwerkranker und sterbender Menschen im Krankenhaus

Die Beteiligung der medizinischen Experten ermöglicht eine Verankerung der Empfehlungen in die klinische Praxis.

\section{Ansprechpartnerin für das Projekt}

Dipl.-Psych. Asita Behzadi

Telefon: 450-553204

Email: asita.behzadi@charite.de

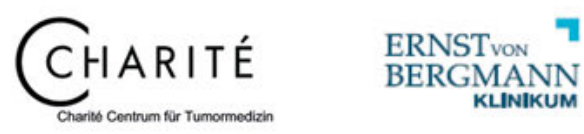


